\title{
Prediction of Biogas Generation Profiles in Wastewater Treatment Plants Using Neural Networks
}

\author{
Peiman Kianmehr, Wathiq Mansoor, and Fadi A. Kfoury
}

\begin{abstract}
The great potential of Waste Activated Sludge (WAS) to produce methane as renewable bio-resource energy has always been of engineers' interest. The evaluation of the rate of methane generation and its ultimate value is a crucial step to predict the performance of anaerobic digesters degrading wide ranges of raw and pre-treated WAS. Biochemical methanogenic potential (BMP) test is known as the most common assay in this context. However, it is known as a time consuming, equipment-intensive and consequently expensive tool. The objectives of this research are to identify key WAS properties required to estimate biodegradability of raw and pretreated sludge and accordingly generate a proper model for predicting sludge biodegradability, utilizing Artificial Neural Networks (ANN). Earlier attempts to identify such key indicators and generating a proper model representing sludge biodegradability using typical mathematical approaches were unsuccessful. However, the results of this research proved ANN effective in modeling sludge biodegradability.
\end{abstract}

Index Terms-Sludge digestion, pre-treatment, solid residence time, artificial neural networks.

\section{INTRODUCTION}

Wastewater is treated using aerobic or anaerobic processes and a considerable fraction of solids in wastewater is separated through physical, chemical or biological treatment processes. These solids are called waste activated sludge (WAS) or biosolids and are generally treated trough aerobic or anaerobic digestion processes. Unlike aerobic processes, the anaerobic processes are known as controlled system emitting low level of gases into the atmosphere and requiring a smaller quantity of energy during treatment processes. The biogas generated through the anaerobic treatment processes consists of $60 \%$ methane which is a combustible gas and an excellent alternative source of energy for non-renewable fossil fuels.

Even though anaerobic digestion processes are more environmentally friendly and energy efficient, they are not as widely used as aerobic processes. This is mainly due to slow process of bioconversion of biosolids to biogas and lack of an entirely practical approach to describing the anaerobic biodegradability of organic materials. These have resulted in a limited and relatively imprecise literature in anaerobic field and leaded engineers to opt aerobic treatment plants as more predictable and accurate solution. Like other parts of the world, such approach is observed in United Arab Emirates

Manuscript received May 15, 2013; revised July 24, 2013.

Peiman Kianmehr, Wathiq Mansoor, and Fadi A. Kfoury are with School of Engineering, American University in Dubai, United Arab Emirates (e-mail: pkianmehr@aud.edu).
(UAE) regardless of the gleaming advantages of anaerobic reactors in producing biogas.

In order to accelerate the bioconversion of biosolids or WAS, the pre-treatment of sludge prior to digestion processes is of increasing interest and has been found as an effective techniques. Pre-treatment technologies have included physical [1], chemical [2] and thermal [3] processes. Ultrasound pre-treatment is a physical pre-treatment technology and was specifically employed in this research. The earlier results revealed that sonication could improve the biodegradation rate of sludge. This was mainly due to considerable solubilization of flocs and particles which results increase in particles' specific surface area and consequently enhancement of the hydrolysis rate which is known as a rate limiting step in anaerobic digestion [4].

One of the most important challenges in anaerobic treatment processes is the biodegradation assessment of organic materials. The biochemical methanogenic potential (BMP) test is used to measure the anaerobic biodegradability potential of wastewater and WAS. The test is conducted in sealed serum bottles. The inoculation of Waste Activated Sludge (WAS) with sludge from an anaerobic digester is typically carried out to provide an initial population of anaerobic bacteria. While performing the test, the generated gas in the headspace is measured daily in the first weeks and occasionally later. The composition of the generated gas is examined to determine the volume of methane and other gases such as $\mathrm{CO}_{2}, \mathrm{H}_{2}$ and $\mathrm{N}_{2}$ [5]. The total methane and carbon dioxide generated in a BMP test is considered to determine the success of methane generation in an anaerobic process and to quantify the impact of pre-treatment technology on sludge biodegradability. The BMP test was found to take about 50-70 days to reach a stable level of accumulated methane. This is due to the low growth rates of methanogenic bacteria in the BMP test as a batch reactor. This makes the assessment process time consuming, expensive and in some cases impractical [2].

A research project has been conducted to investigate the impacts of ultrasound pre-treatment on a wide range of WAS samples. WAS samples with 2, 7 and 15 day of solid residence times (SRTs) generated using three sequencing batch reactors (SBRs) to generate sludge samples under controlled conditions for further investigations. The WAS samples treated by sonication at $45^{\circ} \mathrm{C}$ in a bench scale apparatus in batch mode and ultrasound intensity was evaluated by collecting samples at different times as the treatment progressed. For the unit employed in this study sonication times of 5, 10, 25 and 45 minutes corresponded to ultrasound intensities of $1111,2222,5555$ and $9999 \mathrm{~kJ} / \mathrm{L}$ respectively. The BMP test was conducted for raw (without 
pre-treatment) and pre-treated sample and methane generation was observed for about 50 days. The main data set used in this research has been reflected in Table I and further information regarding the apparatus and the pre-treatment methodology has been provided by Kianmehr et al. [6].

The solubilization of flocs and particles in WAS by sonication is known as the main reason for the enhancement of biodegradation rates and observed accelerated methane generation [4]. The chemical oxygen demand (COD) and total Kjeldahl Nitrogen (TKN) of the sample were measure and they were fractionated into filtered COD (FCOD) and filtered TKN (FTKN). In addition total solids concentration (TS) and volatile dissolved solids (VDS) were measured.
VDS is known as an indicator for organic matters in soluble phase. FCOD/COD and VDS/TS are known as the ratios representing the fraction of organic matters that are solubilized due to sonication and FTKN/TKN is considered as the ratios representing the fraction of organic nitrogen that is solubilized. The experimental results including solubilization indicators that are used in this research are appended to the end of this manuscript. A correlation between these typical solubilization indicators and rate of methane generation are logically expected while such expected correlations were not described by simple mathematical or graphical comparisons.

TABLE I: EXPERIMENTAL RESULTS INCLUDING SOLUBILIZATION INDICATORS

\begin{tabular}{|c|c|c|c|c|c|c|c|c|c|c|c|c|c|c|c|c|c|c|c|c|}
\hline SRT & SI & VDS & FCOD & FTKN & $\begin{array}{c}\text { Time } \\
\text { (Days) }\end{array}$ & BMP & SRT & SI & VDS & FCOD & FTKN & Time (Days) & BMP & SRT & SI & VDS & FCOD & FTKN & \begin{tabular}{|c|} 
Time \\
(Days)
\end{tabular} & BMP \\
\hline \multirow{43}{*}{$\begin{array}{r}1.95 \\
\text { day }\end{array}$} & \multirow{11}{*}{0} & \multirow{11}{*}{6.1} & \multirow{11}{*}{1.3} & \multirow{11}{*}{3.9} & \begin{tabular}{l|}
0 \\
\end{tabular} & 0.00 & & \multirow{11}{*}{0} & \multirow{11}{*}{2.6} & \multirow{11}{*}{1} & \multirow{11}{*}{1.3} & 0 & 0.00 & & \multirow{11}{*}{0} & \multirow{11}{*}{5.8} & \multirow{11}{*}{1.4} & \multirow{11}{*}{1.7} & 0 & 0.16 \\
\hline & & & & & 1 & 0.04 & & & & & & 2 & 0.06 & & & & & & 5 & 0.21 \\
\hline & & & & & 2 & 0.09 & & & & & & 4 & 0.16 & & & & & & 7 & 0.25 \\
\hline & & & & & 4 & 0.16 & & & & & & 6 & 0.23 & & & & & & 11 & 0.27 \\
\hline & & & & & 6 & 0.25 & & & & & & 8 & 0.27 & & & & & & 13 & 0.30 \\
\hline & & & & & 11 & 0.38 & & & & & & 10 & 0.30 & & & & & & 15 & 0.31 \\
\hline & & & & & 16 & 0.42 & & & & & & 13 & 0.32 & & & & & & 20 & 0.32 \\
\hline & & & & & 23 & 0.44 & & & & & & 17 & 0.35 & & & & & & 26 & 0.33 \\
\hline & & & & & 30 & 0.45 & & & & & & 26 & 0.40 & & & & & & 36 & 0.35 \\
\hline & & & & & 46 & 0.45 & & & & & & 31 & 0.42 & & & & & & 43 & 0.35 \\
\hline & & & & & & & & & & & & 54 & 0.43 & & & & & & 57 & 0.36 \\
\hline & \multirow{11}{*}{5} & \multirow{11}{*}{29.9} & \multirow{11}{*}{27.4} & \multirow{11}{*}{48.9} & 0 & 0.00 & & & & & & 0 & 0.00 & & & & & & 3 & 0.19 \\
\hline & & & & & 1 & 0.04 & & & & & & 2 & 0.06 & & & & & & 5 & 0.26 \\
\hline & & & & & 2 & 0.08 & & & & & & 4 & 0.14 & & & & & & 7 & 0.30 \\
\hline & & & & & 4 & 0.13 & & & & & & 6 & 0.21 & & & & & & 11 & 0.31 \\
\hline & & & & & 6 & 0.18 & & & & & & 8 & 0.29 & & & & & & 13 & 0.33 \\
\hline & & & & & 11 & 0.32 & & 5 & 30.5 & 34.5 & 51.2 & 10 & 0.33 & & 10 & 48.1 & 47.2 & 63.8 & 15 & 0.34 \\
\hline & & & & & 16 & 0.43 & & & & & & 13 & 0.36 & & & & & & 20 & 0.35 \\
\hline & & & & & 23 & 0.45 & & & & & & 17 & 0.39 & & & & & & 26 & 0.36 \\
\hline & & & & & 30 & 0.46 & & & & & & 26 & 0.43 & & & & & & 36 & 0.37 \\
\hline & & & & & 46 & 0.47 & 7 & & & & & 31 & 0.44 & & & & & & 43 & 0.37 \\
\hline & & & & & & & day & & & & & 54 & 0.45 & 15 days & & & & & 57 & 0.38 \\
\hline & & & & & 0 & 0.00 & & & & & & 0 & 0.00 & & & & & & 3 & 0.15 \\
\hline & & & & & 1 & 0.03 & & & & & & 2 & 0.07 & & & & & & 5 & 0.18 \\
\hline & & & & & 2 & 0.07 & & & & & & 4 & 0.17 & & & & & & 7 & 0.19 \\
\hline & & & & & 4 & 0.13 & & & & & & 6 & 0.24 & & & & & & 11 & 0.19 \\
\hline & & & & & 6 & 0.19 & & & & & & 8 & 0.32 & & & & & & 13 & 0.20 \\
\hline & 10 & 38 & 39.6 & 63.4 & 11 & 0.33 & & 10 & 43.8 & 43.4 & 65.2 & 10 & 0.35 & & 25 & 56.7 & 60.7 & 80.1 & 15 & 0.20 \\
\hline & & & & & 16 & 0.43 & & & & & & 13 & 0.38 & & & & & & 20 & NA \\
\hline & & & & & 23 & 0.45 & & & & & & 17 & 0.39 & & & & & & 26 & 0.34 \\
\hline & & & & & 30 & 0.46 & & & & & & 26 & 0.43 & & & & & & 36 & 0.36 \\
\hline & & & & & 46 & 0.47 & & & & & & 31 & 0.44 & & & & & & 43 & 0.37 \\
\hline & & & & & & & & & & & & 54 & 0.45 & & & & & & 57 & 0.38 \\
\hline & & & & & 0 & 0.00 & & & & & & 2 & 0.07 & & & & & & 3 & 0.15 \\
\hline & & & & & 1 & 0.03 & & & & & & 4 & 0.17 & & & & & & 5 & 0.18 \\
\hline & & & & & 2 & 0.07 & & & & & & 6 & 0.24 & & & & & & 7 & 0.19 \\
\hline & & & & & 4 & 0.13 & & & & & & 8 & 0.31 & & & & & & 11 & 0.19 \\
\hline & 25 & 420 & 150 & 756 & 11 & 0.36 & & 25 & 54 & 560 & 70. & 10 & 0.34 & & & & & 800 & 13 & 0.20 \\
\hline & 25 & 43.9 & 45.9 & 15.0 & 16 & 0.46 & & 25 & 54.7 & 56.9 & 19.1 & 13 & 0.38 & & 45 & 61.6 & 63.9 & 89.9 & 15 & 0.20 \\
\hline & & & & & 23 & 0.48 & & & & & & 17 & 0.39 & & & & & & 20 & 0.24 \\
\hline & & & & & 30 & 0.49 & & & & & & 26 & 0.43 & & & & & & 26 & 0.34 \\
\hline & & & & & 46 & 0.5 & & & & & & 31 & 0.44 & & & & & & 36 & 0.36 \\
\hline & & & & & & & & & & & & & & & & & & & 43 & 0.37 \\
\hline
\end{tabular}




\section{OBJECTIVE}

The general objective of this research is to develop a practical and accurate tool to predict the anaerobic biodegradability of WAS and the production rate of methane as major renewable bioresource energy without requiring further BMP tests. As the outcome of this project, the significance of each one of these indicators might reveal some details regarding the influence of pre-treatment intensities, sludge properties and solubilization on gas generation.

\section{RESEARCH SCOPE}

\section{A. Data Scope}

The data scopes for the Solid Retention Time (SRT) parameter as well as the Sonication Intensity (SI) used are summarized in Table II. It should be noted that the following research builds upon the data provided by a previous research done by Kianmehr [4] characterizing sonication impacts on methane generation profiles.

TABLE II: SRT AND SI SCOPE

\begin{tabular}{|l|l|}
\hline Parameter & Range \\
\hline Solid Retention Time (SRT) & $1.95-15$ Days \\
\hline $\begin{array}{l}\text { Sonication Duration as indicator of } \\
\text { Intensity (SI) }\end{array}$ & $0-45$ Minutes \\
\hline
\end{tabular}

\section{UTILIZED SOFTWARE}

The utilized software in this research is mainly limited to MatLab R2011a, along with its built-in neural network toolbox. It should be noted that custom MatLab scripts were built for the purpose of creating, training, validating and testing Neural Networks. Further details have been introduced in Table III. Custom MatLab scripts were also used for the purpose of plotting the methane generation profiles of every network created.

TABLE III: NEURAL NETWORK CHARACTERISTICS SUMMARY

\begin{tabular}{|l|l|}
\hline ANN Property & Description \\
\hline Type & Feed-Forward \\
\hline Number of Layers & 4 - Layers \\
\hline Training Algorithm & Levenberg-Marquardt \\
\hline Validation Algorithm & Mean Squared Error \\
\hline
\end{tabular}

\section{RESEARCH Methodology}

The investigation of the methane profiles produced from wastewater treatment plants was based on Artificial Neural Networks (ANN). ANN were utilized in this research due to the highly complex relationships that exist between the four studied parameters (Sludge SRT, VDS, FCOD, and FTKN) and the rate of methane production. One of the targets of this research is to find the best combination of the four studied parameters that would yield to the most accurate predicted methane profiles. The different combinations of parameters tested in this experiment are summarized in Table IV. Feed-Forward back-propagation 4-layer Neural Networks trained based on Levenberg-Marquardt algorithm and validated based on the mean-squared error were utilized in this research. A conservative approach was taken for the testing stage of each of the combinations. For every combination of parameters, different networks were trained each isolating a set of data having the same SRT and SI. The performance of every network was then assessed based on its accuracy in predicting the results of the isolated set.

TABLE IV: COMBINATIONS DESCRIPTIONS

\begin{tabular}{|l|l|}
\hline Combination \# & Description \\
\hline $1-0$ & All Parameters \\
\hline $1-1$ & SRT+SI+VDS+FCOD (FTKN Removed) \\
\hline $1-2$ & SRT+SI+VDS+FTKN (FCOD Removed) \\
\hline $1-3$ & SRT+SI+FCOD+FTKN (VDS Removed) \\
\hline $1-4$ & SRT+SI+VDS (FTKN and FCOD removed) \\
\hline $1-5$ & SRT+SI+FCOD (FTKN and VDS removed) \\
\hline $1-6$ & SI+VDS+FCOD (SRT and FTKN removed) \\
\hline
\end{tabular}

TABLE V: SET \# 2, COMBINATION 1-0 INPUTS, TARGETS, AND ANN PREDICTIONS

\begin{tabular}{|c|c|c|c|c|c|c|c|}
\hline \multicolumn{6}{|c|}{ Inputs } & \multirow{2}{*}{$\begin{array}{l}\text { Predictions } \\
\text { BMP' }\end{array}$} & \multirow{2}{*}{$\begin{array}{l}\text { Targets } \\
\text { BMP }\end{array}$} \\
\hline SRT & SI & VDS & FCOD & FTKN & Days & & \\
\hline \multirow{10}{*}{$\begin{array}{l}1.95 \\
\text { Days }\end{array}$} & \multirow{10}{*}{5} & \multirow{10}{*}{29.9} & \multirow{10}{*}{27.4} & \multirow{10}{*}{48.9} & 0 & 0.04 & 0.00 \\
\hline & & & & & 1 & 0.06 & 0.04 \\
\hline & & & & & 2 & 0.08 & 0.08 \\
\hline & & & & & 4 & 0.12 & 0.13 \\
\hline & & & & & 6 & 0.18 & 0.18 \\
\hline & & & & & 11 & 0.34 & 0.32 \\
\hline & & & & & 16 & 0.43 & 0.43 \\
\hline & & & & & 23 & 0.46 & 0.45 \\
\hline & & & & & 30 & 0.46 & 0.46 \\
\hline & & & & & 46 & 0.47 & 0.47 \\
\hline
\end{tabular}

The neural network trained with combination 1-0 parameters excluding data-set \#2 (1.95 days SRT and $5 \mathrm{~min}$ SI) was chosen to illustrate the research methodology. After creation, the network was tested in predicting the excluded data-set (\#2). Data-set \#2 inputs, targets, and ANN predictions are tabulated in Table $\mathrm{V}$. The predicted profile by the neural network and its regression is shown in Fig. 1 and Fig. 2 respectively. As previously mentioned, the generation of all networks was automated using MatLab custom scripts.

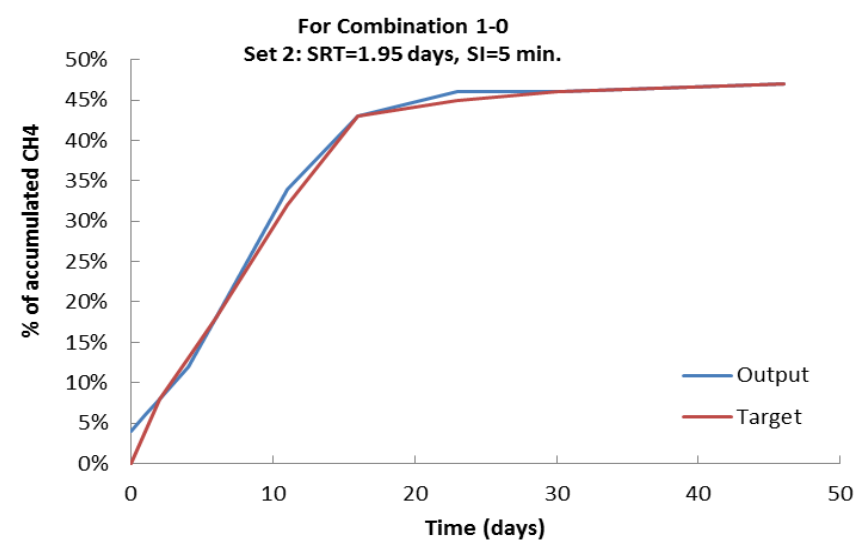

Fig. 1. Sample Predicted-Profile of Combination 1-0 (Simulated on Set \# 2) 


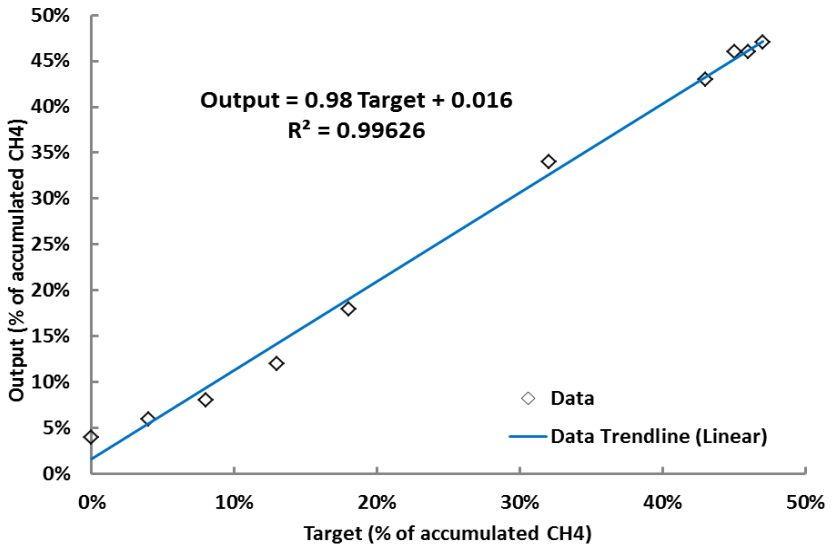

Fig. 2. Sample Regression for Combination 1-0 (Simulated on Set \# 2)

This testing methodology would be one step towards generalizing the prediction capabilities of Neural Networks to SRT's/SI's not covered in the scope of this research.

In nutshell, the standard procedure for training/testing Neural Networks based on random data sets was not utilized in this experiment; however, a more conservative approach that reserved entire data sets having the same SRTs for the purpose of testing the trained networks was adopted. Accordingly, for every combination eight different Neural Networks were generated, each predicting the methane generation profile of the SRT/SI set that was excluded in its training. This allows us to assess whether every sludge sample under certain intensity is predictable or not.

\section{PRELIMINARY RESUltS}

In order to statistically evaluate the performance of every network, a linear regression was performed on the outputs versus the inputs. The slope of the regression line was considered as a preliminary-indicator about the accuracy of predication of every network. The slopes of the regression lines of the generated networks are tabulated in Table VI.

\begin{tabular}{|c|c|c|c|c|c|c|c|c|}
\hline & & \multicolumn{7}{|c|}{ Combination } \\
\hline & & $1-0$ & $1-1$ & $1-2$ & $1-3$ & $1-4$ & $1-5$ & $1-6$ \\
\hline \multirow{8}{*}{ 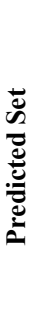 } & 1 & 1.3 & 1.0 & 1.0 & 1.2 & 0.96 & 1.0 & 0.81 \\
\hline & 2 & 0.98 & 0.96 & 1.1 & 1.0 & 0.9 & 1.0 & 0.54 \\
\hline & 3 & 0.59 & 1.1 & 0.92 & 1.1 & 0.93 & 1.0 & 0.40 \\
\hline & 4 & 0.41 & 0.84 & 1.1 & 0.69 & 0.88 & 0.82 & 1.8 \\
\hline & 5 & 0.86 & 1.1 & 1.0 & 0.4 & 0.85 & 0.95 & 0.98 \\
\hline & 6 & 0.77 & 1.0 & 0.91 & 0.93 & 0.89 & 0.99 & 1.2 \\
\hline & 7 & 0.92 & 0.85 & 0.87 & 1.0 & 0.90 & 0.61 & 0.59 \\
\hline & 8 & 0.62 & 0.95 & 0.74 & 0.99 & 0.97 & 0.94 & 0.60 \\
\hline \multicolumn{2}{|c|}{$\begin{array}{l}\text { Reg. } \\
\text { Slope }\end{array}$} & 0.81 & 0.98 & 0.96 & 0.91 & 0.91 & 0.91 & 0.87 \\
\hline \multicolumn{2}{|c|}{$\frac{\text { Std. }}{\text { Deviation }}$} & 0.28 & 0.10 & 0.12 & 0.25 & 0.04 & 0.14 & 0.46 \\
\hline
\end{tabular}

\section{DISCUSSION}

Preliminary results of this research were promising, showing that Neural Networks are indeed capable of predicting the biogas generation profiles of sludge based on the studied parameters. The results also show the importance of the studied parameters in predicting the biogas generation profiles. Table VII lists the combinations following the order of their accuracy in biogas generation profiles prediction.

The results show that the highest accuracy would be achieved by including both the VDS and the FCOD parameters (Combination \# 1-1) within the neural network inputs. The contribution of the FTKN parameter was the most evident when combining it with the VDS parameter.

The combination \# 1-2 having SRT+SI+VDS+ FTKN yielded substantially better accuracy than combination \# 1-4 having SRT+SI+VDS. Conversely, the FTKN parameter when added to the FCOD parameter did not yield to any significant improvement (Combinations \# 1-5 and \# 1-3). Overall, the results show that the FTKN parameter has little contribution in improving the accuracy of the prediction compared to the two main players, the VDS, at the first place, and the FCOD, at the second place.

TABLE VII: ANN INPUT COMBINATIONS SORTED BY THEIR PREDICATION ACCURACY

\begin{tabular}{|c|l|c|}
\hline $\begin{array}{c}\text { Comb. } \\
\text { \# }\end{array}$ & Description & $\begin{array}{c}\text { Average Regression } \\
\text { Slope }\end{array}$ \\
\hline $1-1$ & $\begin{array}{l}\text { SRT+SI+VDS+FCOD (FTKN } \\
\text { Removed) }\end{array}$ & 0.98 \\
\hline $1-2$ & $\begin{array}{l}\text { SRT+SI+VDS+FTKN (FCOD } \\
\text { Removed })\end{array}$ & 0.96 \\
\hline $1-4$ & $\begin{array}{l}\text { SRT+SI+VDS (FTKN and FCOD } \\
\text { removed) }\end{array}$ & 0.91 \\
\hline $1-5$ & $\begin{array}{l}\text { SRT+SI+FCOD (FTKN and VDS } \\
\text { removed) }\end{array}$ & 0.91 \\
\hline $1-3$ & $\begin{array}{l}\text { SRT+SI+FCOD+FTKN (VDS } \\
\text { Removed) }\end{array}$ & 0.91 \\
\hline $1-6$ & $\begin{array}{l}\text { SI+VDS+FCOD (SRT and FTKN } \\
\text { removed })\end{array}$ & 0.81 \\
\hline $1-0$ & All Parameters & \\
\hline
\end{tabular}

The results also reveal that when all the parameters are present together as inputs for a neural network, distorted results were generated. This interference was mainly observed in combination \# 1-0.

Finally, the results also affirmed the great importance of the SRT parameter in enhancing the prediction accuracy of the neural network, as observed in combinations \#1-6 and $\# 1-1$.

Further statistical investigation is currently in progress to acquire deeper insight into the correlations among the indicators.

\section{CONCLUSION}

The following research evaluated the ability of Artificial Neural Networks in predicting the biogas generation potential of Waste Activated Sludge (WAS), given several parameters including: SRT, SI, VDS, FCOD and FTKN. The results revealed that Neural Networks are indeed capable of predicting biogas generation profiles of WAS. In nutshell, the following conclusions could be drawn from the research preliminary-results.

- $\quad$ The best accuracy is achieved when SRT, SI, VDS and FCOD parameters are included as inputs for the neural network.

- The VDS parameter is the major predictor followed 
by the FCOD parameter when SRT and SI values are known.

- The FTKN parameter has little contribution in enhancing the neural network prediction accuracy.

- Distorted prediction results could be obtained from a neural network having excess input parameters.

- $\quad$ The SRT parameter is a major parameter that cannot be excluded, even if both VDS and FCOD parameters were included.

\section{REFERENCES}

[1] E. Cartmell, S. Clay, R. Smith, and S. Sithey, "Application of Mechanical Pretreatments for Improving the Digestibility of Waste Activated Sludge," in Proc. 10th World Congress on Anaerobic Digestion, Montreal, Quebec, 2004,

[2] P. Kianmehr, W. Parker, and P. Seto, "An evaluation of protocols for characterization of ozone impacts on WAS properties and digestibility," Bioresource Technology, vol. 101, no 22, pp. 8565-8572, 2010.

[3] B. Park, J. H. Ahn, J. Kim, and S. Hwang, "Use of microwave pretreatment for enhanced anaerobiosis of secondary sludge," Water Science and Technology, vol. 50, no. 9, pp. 17-23, 2004.

[4] P. Kianmehr, "Characterization of pretreatment impacts on WAS properties and digestibility", Ph.D. thesis, Univ. of Waterloo, Waterloo, Canada, 2010.
[5] J. Straub, A. C. Conklin, J. F. Ferguson and H. D. Stensel, "Use of the ADM1 to investigate the effects of acetoclastic methanogen population dynamics on mesophilic digester stability," Water Sci. Technol., vol. 54, no. 4, pp. 59-66, 2006.

[6] P. Kianmehr, W. Parker, and P. Seto, "Assessment of WAS pretreatment by ultrasound and applicability of biodegradability indicators," Journal of Environmental Engineering (ASCE), voll. 139, no. 4, 545-553, 2013.

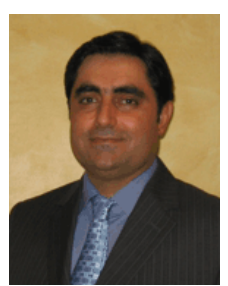

Peiman Kianmehr's expertise is in water and environmental engineering, with emphasis on water \& wastewater treatment and environmental chemistry. His research work involves physical, biochemical and biological characterization of waste activated sludge to identify pretreatment ozonation and sonication impacts. Prior to joining AUD, he worked with Environment Canada on the design, operation and evaluation of aerobic and anaerobic reactors as well as pretreatment (ozonation and sonication) reactors. Between 1999 and 2004, he directed various design, planning, and QC studies in the field of hydraulic engineering, and worked on major water transmission and supply projects, as well as earth dam construction and flood control projects. Peiman is a member of the Water Environment Federation (WEF) and the Water Environment Association of Ontario (WEAO) and is a regular participant at the WEFTEC Conferences. 